\title{
Mesure du flux de sève brute dans le tronc du Douglas par une nouvelle méthode thermique
}

\author{
A. GRANIER \\ avec la collaboration technique de P. GROSS \\ INRA, Station de Sylviculture et de production, Centre de Recherches de Nancy \\ Champenoux, F 54280 Seichamps
}

\begin{abstract}
Résumé
Une nouvelle méthode de mesure du flux de sève brute dans le tronc est utilisée ici pour étudier les transferts hydriques chez le Douglas. Les variations azimutales du flux de sève ont été mesurées pendant 23 jours selon trois orientations et n'ont pas montré de forte hétérogénéité des transferts d'eau. Les variations verticales du flux de sève ont ensuite été analysées à deux hauteurs : base du tronc et base de la couronne vivante. A ce niveau $(8 \mathrm{~m})$, si la densité de flux est toujours supérieure à celle de la base du tronc, les flux totaux sont sensiblement égaux pour un arbre bien alimenté en eau. Un dessèchement imposé à l'arbre fait apparaître progressivement un écart de flux entre les deux hauteurs mesurées, correspondant à une déplétion du "réservoirtronc ", d'environ 28 litres, soit $4,2 \mathrm{~mm}$. La réhydratation met en évidence deux phases : une réponse rapide de l'arbre qui débute quelques minutes après le début de l'apport d'eau : il s'agit de la recharge partielle des tissus élastiques de l'arbre. La deuxième phase correspond à la réhydratation progressive du bois d'aubier du tronc et dure environ 15 jours.
\end{abstract}

Mots clés: Pseudotsuga menziesii, flux de sève, sécheresse, xylème, stockage hydrique.

\section{Introduction}

Parmi les différents moyens d'estimer la consommation en eau par les arbres adultes, la mesure du flux de sève brute est largement utilisée. Ces méthodes permettent des études à des échelles de temps et d'espace souvent assez fines. Une des plus anciennes (HUBER \& SCHMIDT, 1937), la méthode des impulsions de chaleur, reste une des plus utilisée grâce à son faible coût et au peu de traumatisme imposé à l'arbre. Il existe toutefois deux limitations essentielles à l'utilisation de cette méthode :

- les mesures sont ponctuelles, alors que le flux de sève varie avec la profondeur dans le xylème (Swanson, 1967 et 1974);

- son imprécision est d'autant plus grande que le flux de sève est faible.

Nous avons proposé récemment (Granier, 1985) une nouvelle méthode basée sur un capteur thermique à chauffage continu qui intègre la mesure du flux de sève brute suivant un axe radial dans le xylème du tronc, et qui permet d'estimer avec précision des flux hydriques faibles. 
L'objectif de cette étude est d'analyser au moyen de cette méthode les caractéristiques du transfert d'eau dans des Douglas adultes en conditions naturelles. En effet, peu de recherches permettent actuellement de connaître quelles sont les variations des transferts hydriques dans les arbres adultes: signalons toutefois sur le Douglas, le travail récent de CoHEN et al. (1985) sur les distributions radiale et azimuthale du phénomène.

Un aspect essentiel de la dynamique des transferts d'eau dans les arbres adultes réside dans le rôle du tronc. Lieu privilégié de circulation de sève brute, le tronc est aussi considéré comme une structure d'échanges réversibles d'eau avec le flux principal. Dans le but de quantifier ce phénomène, nous analysons ici l'évolution des flux mesurés aux deux extrémités du tronc d'un Douglas soumis à un dessèchement.

\section{Matériel et méthodes}

\subsection{Dispositif expérimental}

L'étude a été réalisée en forêt d'Amance, près de Nancy, dans une plantation de Douglas âgés de 24 ans. Cette plantation est établie sur un sol brun faiblement lessivé, développé sur des marnes du Lias. Les horizons superficiels (de 0 à $60 \mathrm{~cm}$ ) présentent un taux de limons élevés ( 40 p. 100 de limons grossiers et 20 p. 100 de limons fins), et environ 25 p. 100 d'argile. Au-dessous de $60 \mathrm{~cm}$ environ apparaît un horizon de pseudogley, avec un taux d'argile de 35 p. 100. Dans la parcelle expérimentale. de densité 1384 tiges/ha, ont été construits deux lysimètres, chacun autour de deux arbres ; ils sont constitués d'une paroi en beton, doublée d'un film plastique, coulée dans une tranchée de $1,2 \mathrm{~m}$ de profondeur. Le fond de ces lysimètres n'est pas clos. La présence entre 60 et $70 \mathrm{~cm}$ de profondeur, de la couche de pseudogley, limite les échanges d'eau dans le sens vertical. Un toit en matière plastique rigide amovible permet de supprimer l'arrivée des précipitations, et des colliers spéciaux, l'écoulement de l'eau autour des troncs.

\subsection{Mesures de flux de sève}

Nous rappelons brièvement la méthode utilisée, décrite dans un précédent article (Granier, 1985).

Un capteur de mesure du flux de sève est constitué de deux sondes cylindriques de $2 \mathrm{~cm}$ de longueur et $2 \mathrm{~mm}$ de diamètre, insérées radialement dans le bois d'aubier du tronc, et séparées d'une distance de $10 \mathrm{~cm}$ environ. Une de ces sondes (la plus haute dans le tronc) renferme un enroulement chauffant en constantan (voir photo 1) ; elle est chauffée à puissance constante $(0,200 \mathrm{Watt})$. Chaque sonde contient à mi-longueur un thermocouple cuivre-constantan. Les deux thermocouples sont montés en opposition, ce qui permet de mesurer l'écart de tempéralure entre les deux sondes. Un cylindre en aluminium recouvre chaque sonde pour en uniformiser la température. En l'absence de flux de sève, un écart de température $\Delta \mathrm{TM}$ maximum s'établit entre les deux sondes. Lorsqu'il y a flux de sève, une partie de la chaleur dégagée par l'élément chauffant est évacuée par convection; l'écart de température s'établit à une valeur $\Delta \mathrm{T}$ (u) qui 




Pното 1

La sonde chaude du fluxmètre radial.

The heated probe of the radial flowmeter.

dépend de la densité de flux de sève u au voisinage de la sonde. Des étalonnages ont conduit à une relation indépendante du type de bois étudié :

$$
\begin{gathered}
\mathrm{k}=\frac{\Delta \mathrm{TM}-\Delta \mathrm{T}(\mathrm{u})}{\Delta \mathrm{T}(\mathrm{u})}=0,0206 \mathrm{u}^{0,8124} \\
\mathrm{u} \text { en } 10^{-6} \text { m.s. }^{-1}
\end{gathered}
$$

$\mathrm{k}$ représente un nombre sans dimension, qui ne dépend que de $\mathrm{u}$.

L'estimation du terme $\Delta \mathrm{TM}$, essentiel pour le calcul de u, est réalisé pour chaque sonde en prenant l'enveloppe supérieure de la succession des maximas nocturnes sur une période de mesure de l'ordre de 10 jours. En effet, les conditions climatiques, notamment en été, sont parfois favorables à une transpiration nocturne, ce qui se traduit par un $\Delta \mathrm{T}(\mathrm{u})$ non maximal.

Le flux de sève total $F$ traversant le tronc est calculé à partir de u et de la section transversale de bois d'aubier SA au niveau de mesure par:

$$
\mathrm{F}=\mathrm{u} \cdot \mathrm{SA}
$$

Soit d'après (1)

$$
\mathrm{F}=118,99 \cdot 10^{-6} \cdot \mathrm{k}^{1.231} \cdot \mathrm{SA}
$$

avec $\mathrm{SA}$ en $\mathrm{m}^{2}$

$$
\text { F en } \mathrm{m} . \mathrm{s}^{-1}
$$

SA est estimé à partir de prélèvements de carottes de bois dans le tronc. Chez le Douglas, le bois d'aubier se distingue très facilement du bois de cœur par son aspect translucide. L'erreur maximum dans l'estimation de SA par la mesure sur une seule carotte est d'environ 10 p. 100. 


\subsection{Protocole expérimental}

Chacun des quatre arbres a été équipé d'un capteur de flux de sève dans le tronc à $1 \mathrm{~m}$ de hauteur environ. Un cinquième capteur a été placé à la base de la couronne vivante du plus gros arbre du lysimètre où sera appliquée une sécheresse. Ce capteur est situé à une hauteur de $8 \mathrm{~m}$ au-dessus du sol. Le toit amovible de ce lysimètre (traitement "sec») a été mis en place le 27 juin au moment d'une irrigation, et le dessèchement s'est prolongé jusqu'au 8 août, date d'une irrigation.

Un microdendromètre automatique a été installé sur chacun des 4 arbres à $1,3 \mathrm{~m}$ de hauteur ; ces appareils permettent de mesurer des variations de circonférence avec une précision de $0,05 \mathrm{~mm}$.

La saisie automatique du signal des capteurs de flux de sève et de croissance a été assurée à une cadence horaire du 29 mai au 22 septembre 1984.

Enfin, les paramètres climatiques sont mesurés grâce à un poste météorologique automatique situé à proximité $(500 \mathrm{~m})$ de la parcelle. Température, humidité, rayonnement global, pluie et vitesse du vent sont enregistrés chaque minute avec un bilan horaire.

\section{Résultats}

\subsection{Variabilité du flux de sève bruie dans le tronc selon lazimut}

Une expérience préliminaire a consisté à etudier la variabilité du flux de sève dans le tronc suivant différents azimuts de la même hauteur $(1 \mathrm{~m})$. Trois capteurs ont été insérés sur des axes formant entre eux des angles de $120^{\circ}$, et les mesures ont été faites pendant 23 jours consécutifs. La figure la reporte l'évolution du flux de sève journalier pour cette période d'étude, selon les trois orientations dans le tronc (nord-ouest, nordest et sud). On peut noter une assez grande homogénéité des mesures, notamment pour des journées où le flux de sève est faible. Un écart systématique apparaît en condition de flux hydrique plus important, ici au-delà de 8 litres/jour pour cet arbre. La figure $1 \mathrm{~b}$ représente l'évolution du flux de sève selon les trois axes pour une journée à faible demande climatique (E.T.P. ${ }^{(1)}=2,13 \mathrm{~mm}$ ); les mesures de flux des trois capteurs restent très voisines tout au long de la journée. La figure lc représente le cas d'une journée où la demande climatique est plus élevée $($ E.T.P. $=4,07 \mathrm{~mm}$ ); des différences non négligeables apparaissent aux heures de forte transpiration.

Pour cette journée, si la moyenne des valeurs fournies par trois sondes permet d'estimer le flux total à 10,8 litres d'eau, les mesures individuelles prédisent $10,8,11,4$ et 10,2 litres, soit un écart relatif de $6 \mathrm{p}$. 100 autour de la moyenne. Lassore et al. (1977), puis Cohen et al. (1985), à partir de mesures de vitesse des impulsions de chaleur ont aussi pu mettre en évidence de faibles écarts de flux selon l'orientation dans le tronc.

(1) E.T.P. = Evapotranspiration potenticlle (calculée au moyen de la formule de Penman). 

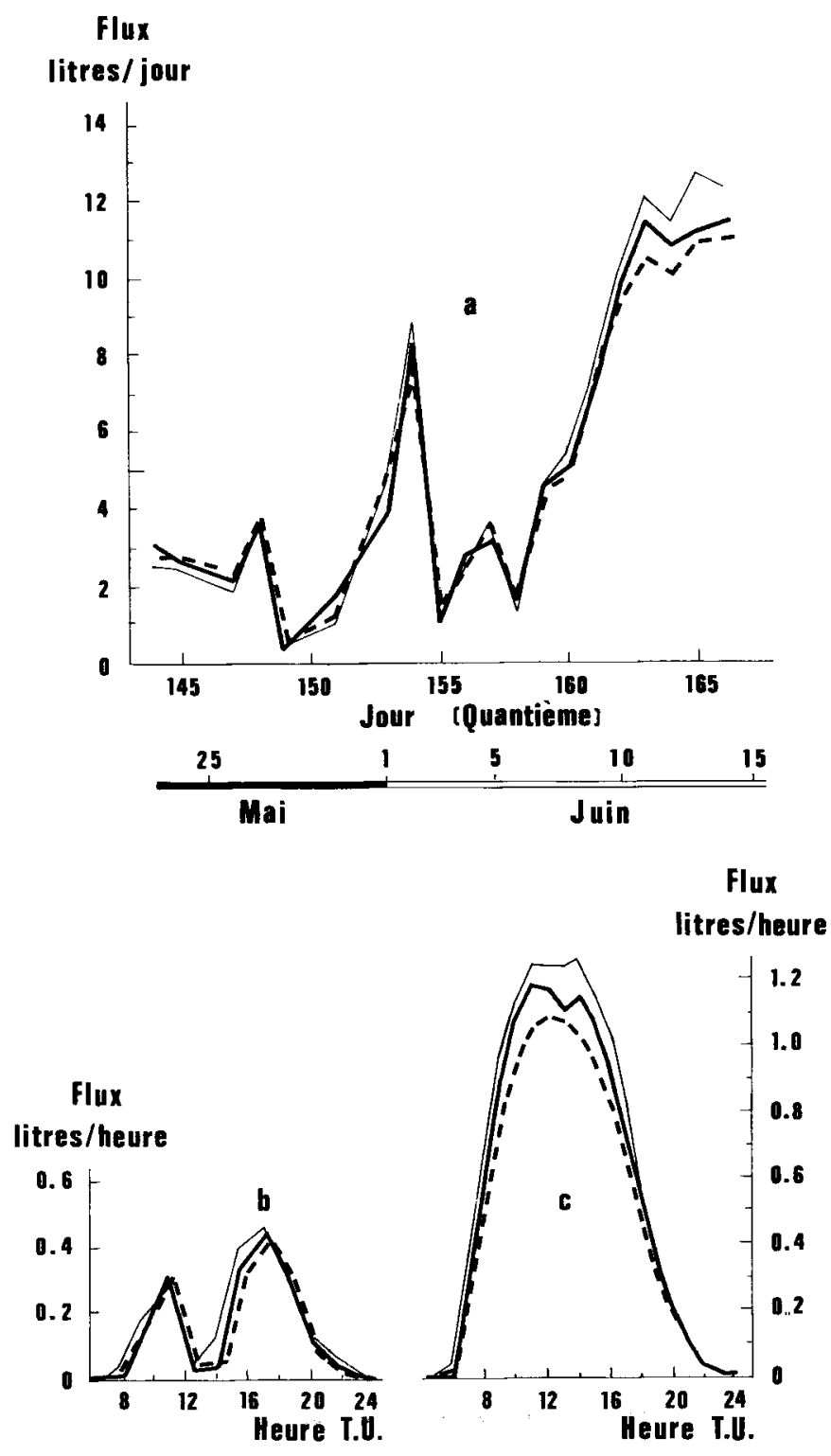

Fig. 1

Variation du flux de sève mesuré selon trois azimuts dans le tronc d'un Douglas. Variation of sap flow in a Douglas-fir tree trunk along 3 different axis.

a : Flux journalier, du 23-05 au 13-06-1984. - Variation from 05-23 to 06-13-1984.

b : Le 23-05-1984 : journée couverte avec pluie. - Cloudy day with a rain (05-23-1984).

c: Le 12-06-1984 : journée ensoleillée. - Sunny day (06-12-1984).

Axe Nord-Ouest. - North-West.

Axe Sud. - South.

Axe Nord-Est. - North-East. 
Il est toutefois difficile de dissocier la variabilité due à l'hétérogénéité des transferts dans le tronc de celle liée à la technique de mesiure. La réponse des capteurs dépend en effet :

- des caractéristiques d'échange de la chaleur au niveau de leur élément chauffant, donc de la qualité du contact thermique qu'ils ont avec le tissu conducteur ;

- de l'épaisseur de bois d'aubier au niveau de la sonde chauffante, qui peut différer selon les trois rayons mesurés dans cette expérience. Signalons toutefois que la largeur de bois d'aubier au niveau des capteurs est de $30 \pm 3 \mathrm{~mm}$; la longueur utile des sondes étant de $20 \mathrm{~mm}$, celles-ci sont intégralement incluses dans une zone conduisant l'eau.

\subsection{Comparaison du flux de sève à deux hauteurs dans le tronc}

Deux niveaux de mesure ont été choisis sur la même génératrice d'un des deux arbres du lysimètre soumis au dessèchement. Le niveau le plus élevé est situé à $8 \mathrm{~m}$ de hauteur, juste au-dessous de la couronne vivante, le plus bas à $1 \mathrm{~m}$ de hauteur. Les deux capteurs de flux limitent ainsi un volume de tronc sans branches d'une longueur de $7 \mathrm{~m}$ et de diamètres $20,6 \mathrm{~cm}$ et $15.3 \mathrm{~cm}$ pour les niveaux bas et haut respectivement. Compte tenu de la décroissance régulière de la section de bois d'aubier avec la hauteur dans le tronc (Granier, 1981), la section conductrice de l'eau aux deux niveaux de mesure n'est pas la même : l'estimation de ce's sections par prélèvements de carottes est de $160 \mathrm{~cm}^{2}$ et $99 \mathrm{~cm}^{2}$ environ pour les niveaux bas et haut. La figure 2a représente pour une journée (le 25-8-1984), la densité de flux exprimée en litres par $\mathrm{dm}^{2}$ de bois d'aubier et par heure pour chacun des deux niveaux de mesure. On constate que la densité de flux hydrique est à toute heure de la journée plus importante en haut qu'en bas du tronc. On peut vérifier sur la figure $2 b$ que les flux totaux, calculés pour les deux niveaux (cf. équation (2)), sont du même ordre de grandeur tout au long de la journée. Néanmoins, les flux de sève aux deux niveaux ne sont pas toujours rigoureusement égaux et ces écarts peuvent avoir plusieurs origines:

(1) l'erreur dans l'estimation de la section de bois d'aubier, qui peut introduire un biais systématique entre les deux mesures de flux :

(2) comme il a été vu précédemment, la mesure du flux de sève suivant un axe radial peut représenter de façon imparfaite le flux total, notamment à certaines heures de la journée ;

(3) enfin, une autre cause de l'écart entre les flux entrant et sortant du tronc réside dans le caractère non conservatif du flux de sève. Dans l'exemple de la figure $2 \mathrm{~b}$, on peut constater que de $8 \mathrm{~h}$ à $12 \mathrm{~h}$ TU, le flux de sève mesuré à la base de la couronne vivante est supérieur à celui qui circule en bas du tronc, ce qui permet de conclure à un dessèchement de la portion de tige située entre les deux capteurs. Cette observation est cohérente avec le modèle proposé par KATERI et al. (1983), pour une culture herbacée, qui considère un réservoir placé en dérivation du trajet hydrique principal; ce réservoir se vide le matin jusqu'en début d'après-midi et se recharge le soir. Toutefois, le fonctionnement hydrique du tronc de l'arbre semble plus complexe, car le dessèchement du tronc (de $8 \mathrm{~h}$ à $12 \mathrm{~h}$, figure 2b) semble suivi d'une phase de réhydratation (de $16 \mathrm{~h}$ à $22 \mathrm{~h}$ ). Il est actuellement difficile de proposer dans notre cas un modèle de fonctionnement au niveau journalier, compte tenu des sources d'incertitude (1) et (2). 

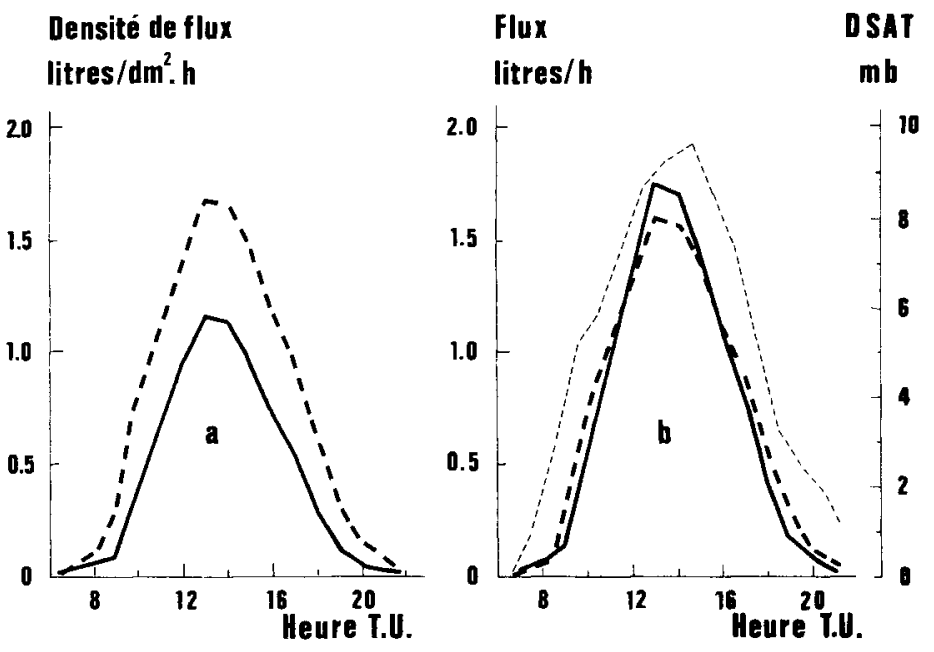

FiG. 2

Evolution journalière (le 25-08-1984) du flux de sève mesuré à deux niveaux de hauteur dans le tronc d'un Douglas.

Diurnal variation of sap flow within a Douglas-fir tree trunk for two heights (08-25-1984).

a : Densité de flux hydrique. - Sap flow density.

b : Flux total. - Total sap flow.

Hauteur $1 \mathrm{~m}$. - Height $1 \mathrm{~m}$.

Hauteur $8 \mathrm{~m}$. - Height $8 \mathrm{~m}$.

Déficit de saturation de l'air. - Vapour deficit of saturation.

\subsection{Transferts hydriques en phase de dessèchement et de réhydratation}

Les apports d'eau ont été arrêtés le 27/06/84. Mais, partant d'un état hydrique du sol proche de la capacité au champ, les effets du dessèchement n'ont été sensibles qu'à partir du 19/07/84. La figure 3 reporte l'évolution du flux de sève (cumuls journaliers) et d'un index qui est une estimation de la transpiration maximale pour l'arbre étudié. Cet index est calculé à partir de la moyenne journalière du déficit de saturation de l'air (DSAT) et du rayonnement global (RG). Nous avons utilisé la formule :

$$
\begin{aligned}
\mathrm{T}= & \mathrm{a} \cdot \frac{\mathrm{DSAT}}{\mathrm{DSAT}+\mathrm{b}} \cdot \frac{\mathrm{RG}}{\mathrm{RG}+\mathrm{c}} \\
& \mathrm{DSAT} \text { en } \mathrm{mb} \\
& \mathrm{RG} \text { en } \mathrm{J} \mathrm{cm}^{-2}
\end{aligned}
$$

utilisée par Lohammar (in HaLldin et al., 1980) pour modéliser les variations de résistance stomatique dans un peuplement de résineux. Les coefficients $a, b, c$ ont été estimés par régression non linéaire entre le flux journalier, DSAT et RG sur des journées où l'arbre a été bien alimenté en eau. Le caractère non limitant du fonctionnement hydrique de l'arbre a été jugé d'après l'examen des courbes de croissance en circonférence journalières ; il a pu être montré (Aussenac et al., 1984) qu'un des premiers indices de l'apparition du stress hydrique était l'arrêt de la croissance en 
circonférence, suivi d'une rétraction du tronc; les journées utilisées pour estimer les trois coefficients de l'équation (4) ont été choisies avant l'apparition de ces phénomènes. Nous avons obtenu les valeurs : $\mathrm{a}=58,5, \mathrm{~b}=6,32, \mathrm{c}=687$.

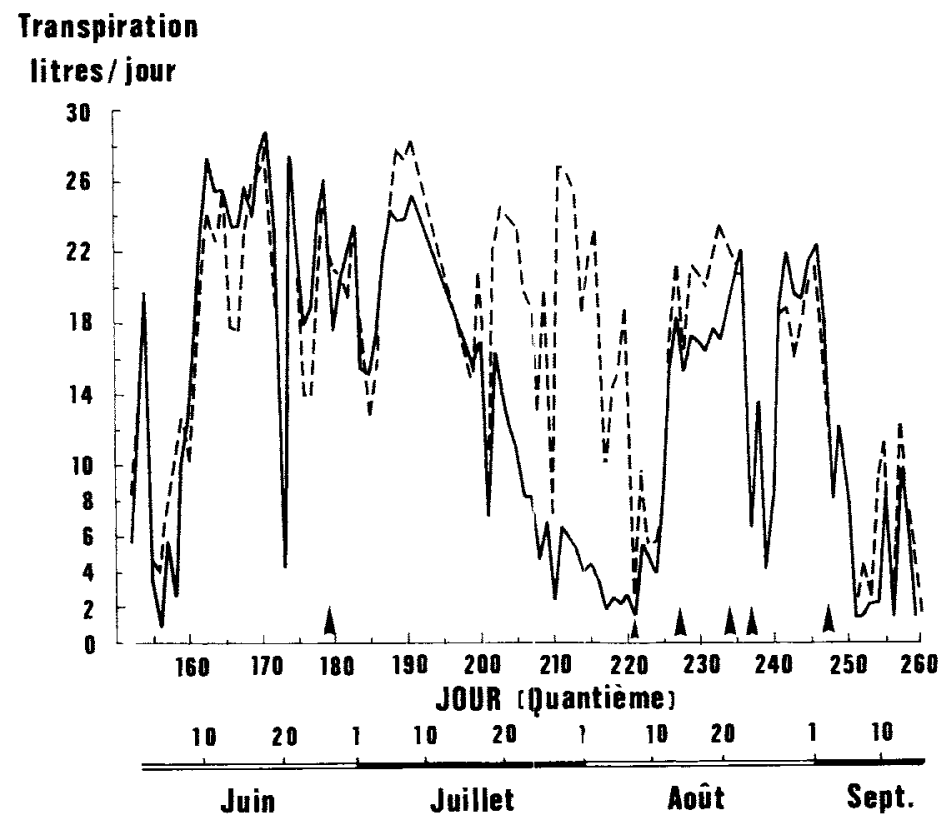

Fig. 3

Evolution saisonnière du flux de sève pour un Douglas en lysimètre. En pointillé, évolution de la transpiration potentielle (voir texte). Les flèches indiquent les dates des différents apports d'eau.

Seasonal variation of sap flow measured for a Douglas-fir tree enclosed in a lysimeter. Dashed line describes potential transpiration (defined in the text).

Dates of watering are shown by arrows.

L'effet de la sécheresse sur la diminution de la transpiration est très brutal : sur la figure 3 , la divergence entre les mesures et le modèle marquent les périodes où il y a chute de transpiration de l'arbre. Les mesures d'humidité du sol pendant cette période ont permis de montrer que le début de la chute de transpiration du Douglas apparaît à un niveau de sécheresse du sol peu important: au jour 201, l'humidité volumique moyenne des 60 premiers cm est de 24 p. 100, ce qui représente un potentiel hydrique moyen de l'ordre de $-0,15 \mathrm{Mpa}$.

Dans le précédent paragraphe, pour une alimentation hydrique de l'arbre satisfaisante, nous avions constaté peu de différences entre les flux aux deux niveaux de mesure. Or, l'examen de la figure 4, qui représente l'évolution du flux de sève aux deux niveaux dans le tronc met en évidence, au niveau des cumuls journaliers, un écart parfois important entre les mesures de flux de sève à $1 \mathrm{~m}$ et à $8 \mathrm{~m}$ de hauteur dans le tronc. Pour mieux étudier ce phénomène, nous avons calculé, sur l'ensemble des 


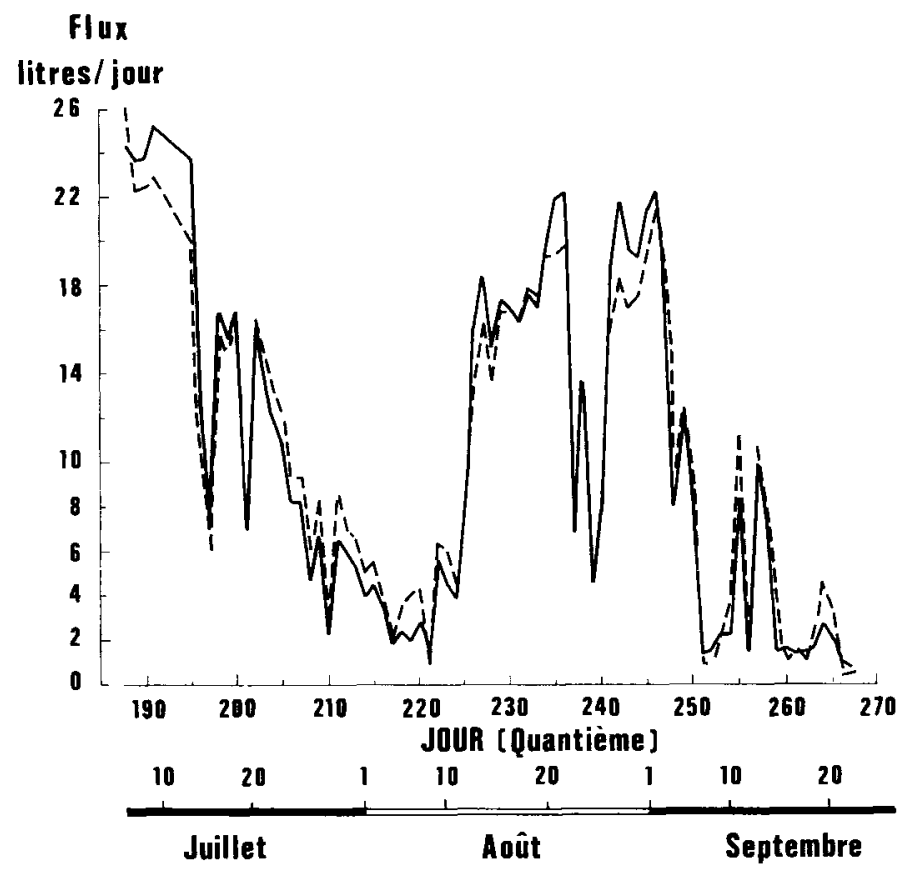

FlG. 4

Evolution saisonnière du flux de sève mesuré à deux niveaux de hauteur dans le tronc pour un Douglas en lysimètre.

Seasonal variations of sap flow at two vertical locations within a Douglas-fir tree enclosed in a lysimeter.

Hauteur $1 \mathrm{~m}$. - Height $1 \mathrm{~m}$.

Hauteur $8 \mathrm{~m}$. - Height $8 \mathrm{~m}$.

journées de mesure, la somme des écarts (S) entre les flux en bas (FB) et en haut du tronc $(\mathrm{FH})$ :

$$
\sum_{i} \mathrm{~S}=\sum_{i}(\mathrm{FB}-\mathrm{FH})
$$

Cette variable représente le stock d'eau échangé par le tronc de l'arbre. Quand $\Sigma S$ diminue, cela signifie une perte d'eau et lorsque $\Sigma S$ augmente, il y a réhydratation du tronc.

La figure 5 reporte l'évolution en fonction du temps de $\Sigma S$. On constate une diminution relativement régulière du stock d'eau dans le tronc au cours du dessèchement : pendant cette phase, l'eau du bois d'aubier du tronc est cédée dans le flux de transpiration. Au moment de la plus forte décroissance du stock d'eau du tronc, on note un assèchement de l'ordre de 2 litres par jour, soit un équivalent en eau de $0,3 \mathrm{~mm}$ par jour. L'écart extrême entre la fin du dessèchement et le moment où la réhydratation est maximale, atteint 28 litres, soit $4,2 \mathrm{~mm}$ d'eau pour la fraction du tronc comprise entre les deux niveaux de mesure. Une estimation, basée sur la quantité 


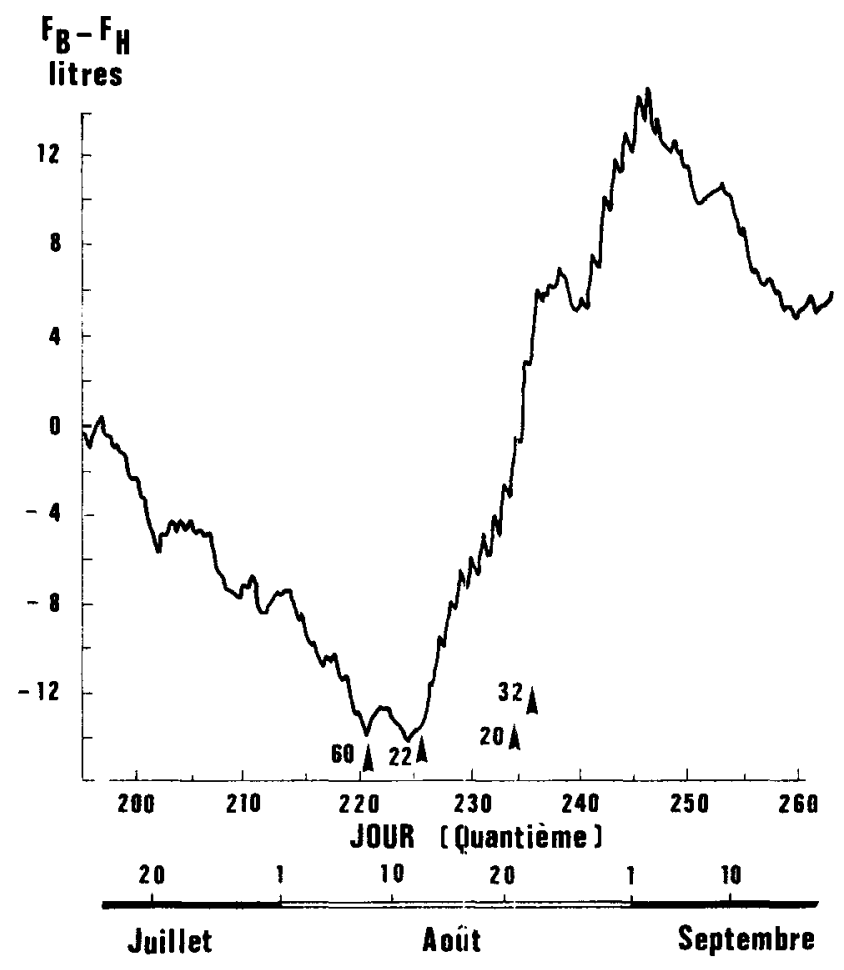

FiG. 5

Variation du stock d'eau dans le tronc d'un Douglas (voir texte) lors d'un cycle de dessèchement-réhumectation. Les flèches verticales indiquent les dates des apports d'eau et leurs quantités sont exprimées en $\mathrm{mm}$.

Effect of soil dehydratation and watering on water reserves within a Douglas-fir tree trunk. Periods of watering are shown by arrows, water quantities are expressed in $\mathrm{mm}$.

relative de bois d'aubier dans le tronc et les branches, permet d'avancer la valeur de 41 litres, soit $6,1 \mathrm{~mm}$ d'eau échangée sur l'ensemble de l'arbre étudié. Le niveau de sécheresse atteint, lors de cette expérience, n'est pas très important: le potentiel hydrique de base est descendu jusqu'à - 1.25 MPa. Waring et RunNing (1978) ont effectué des prélèvements périodiques de bois d'aubier dans des troncs de Douglas en peuplement. Ils ont observé des fluctuations estivales de la quantité d'eau du tronc de l'ordre de $7 \mathrm{~mm}$ correspondant à des séquences de dessèchement-réhumectation.

On constate enfin, sur la figure 4 que les conditions de transfert hydrique dans le tronc, ne correspondent que rarement à un régime de flux conservatif.

La réhydratation, pour ramener le sol à la capacité au champ a été réalisée en plusieurs fois. Le premier apport d'eau a êté effectué lors d'une journée très pluvieuse (le $08 / 08 / 84$ ) sous forme d'une irrigation brutale de $60 \mathrm{~mm}$ d'eau. Du point de vue des transferts d'eau, deux phases sont à distinguer: 


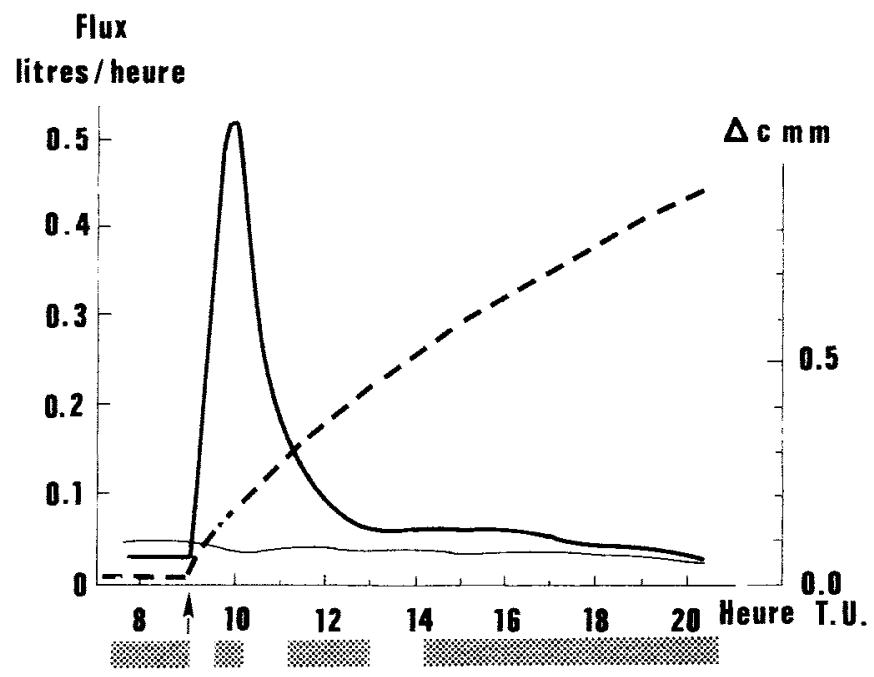

FIG. 6

Evolution du flux de sève (__ et de la circonférence $\Delta C$ (_- $\left._{-}\right)$d'un Douglas irrigué après une période de dessèchement. La flèche verticale indique le début de l'irrigation.

En comparaison, flux de sève d'un Douglas maintenu à la capacité du champ (

Sap flow trends (__ ) and evolution of diameter (___- ) for a Douglas-fir tree submitted to watering after a drought. Beginning of irrigation is shown by an arrow.

(_) : sap flow of a Douglas-fir maintained at field capacity.

Pluie - Rain

- Une phase de réponse rapide de l'arbre, qui débute quelques minutes $(6$ à $8 \mathrm{mn}$ ) après le début de l'apport d'eau. La figure 6 représente, pour la journée de l'irrigation, l'évolution du flux de sève à la base du tronc pour le Douglas irrigué, précédemment desséché et pour un arbre témoin, toujours bien arrosé, situé dans un deuxième lysimètre. On observe une montée rapide de sève brute dans le tronc, avec un maximum de flux situé environ une heure après l'apport d'eau; le flux diminue ensuite progressivement au cours de la journée. Le choix d'une période pluvieuse nous permettant d'affirmer qu'il n'y a pas eu de sortie d'eau au niveau du feuillage, le flux d'eau mesuré correspond à une réhydratation de l'arbre. Le calcul conduit à une quantité d'eau de 1,98 litre. Des mesures de circonférence au moyen de microdendromètres automatiques montrent que cette phase de réhydratation du tronc coïncide avec un brutal accroissement de la circonférence, de l'ordre de $0,5 \mathrm{~mm}$ en 5 heures. L'eau stockée pour l'arbre semble ainsi, en partie, utilisée dans le tronc pour réhydrater ses tissus élastiques. Jarvis (1975), distingue dans le tronc, deux catégories de tissus échangeurs d'eau : les tissus vivants (cambium + phloème) et les tissus " non vivants * (bois d'aubier); c'est la réhydratation des premiers que nous mettons ici en évidence.

- Une phase de réponse de l'arbre à plus long terme, qui correspond à la réhydratation progressive du bois daubier du tronc. En fait, dans notre expérience, nous avons apporté de l'eau en plusieurs fois et l'on peut constater, sur la figure 5 , qu'il y a un délai marqué entre les apports d'eau et la réhydratation du tronc (4 à 5 jours). Enfin, la transpiration ne revient à son niveau optimal que 15 jours après l'irrigation comme il apparaît sur la figure 3. 


\subsection{Discussion et conclusion}

Les mesures de flux de sève selon différents azimuts ont permis de montrer l'existence d'une hétérogénéité des transferts d'eau dans le bois d'aubier du tronc. La sectorisation des échanges hydriques dans les troncs mise en évidence par GreEnidge (1957), Postlethwait \& Rogers (1958), et Vite \& Rudinsky (1959), permet d'expliquer en partie tout au moins cette hétérogénéité. Dans les cas les plus marqués, un secteur déterminé communiquerait avec une portion de la couronne de l'arbre. Ce phénomène reste toutefois limité par le Douglas, et certainement chez les résineux, dont le bois d'aubier est caractérisé par une structure conductrice homogène composée essentiellement de trachéides, éléments dans lesquels les possibilités de transfert hydrique latéral sont importantes, et permettent cl'uniformiser le flux de sève.

L'étude de la phase de dessèchement des arbres a permis de montrer qu'au-delà d'un seuil de potentiel hydrique du sol d'environ $0,15 \mathrm{MPa}$, la transpiration est brutalement affectée. Il est intéressant de constater que c'est à partir du même niveau de sécheresse que se produit l'arrêt de croissance en circonférence du tronc, puis une contraction, résultat reporté lors d'une étude antérieure dans le même dispositif expérimental (Aussenac et al., op. cit.).

Lorsque s’installe la sécheresse, nous avons pu mettre en évidence des écarts importants entre les flux aux deux extrémités d'une portion de tronc. Bien que le cumul des écarts entre les flux $(\Sigma S)$ puisse présenter un biais non négligeable à cause de l'erreur dans l'estimation des sections de bois d'aubier aux deux niveaux de mesure, l'ordre de grandeur de la quantité d'eau échangée est cohérent avec les résultats d'autres auteurs (WARING \& RUNNING, 1978).

L'analyse de ces mesures de flux de sève dans le tronc d'un arbre montrent la complexité des échanges d'eau, étroitement liés à l'état hydrique de l'arbre et à la demande transpiratoire. Il apparaît que du point de vue des phénomènes de stockage et restitution d'eau, l'on puisse distinguer deux types de réservoirs :

- l'un formé par les tissus élastiques de l'arbre, de faible capacité d'échange $(0,3$ à $0,5 \mathrm{~mm}$ d'eau), mais à constante de temps brève (échanges rapides). Ce réservoir est sollicité tout au long de la journée en fonction de la demande climatique, comme l'attestent les variations diurnes de la circonférence (Lassole, 1979 ; Aussenac et al., 1982) :

- le deuxième réservoir, qui compose la plus grande part de la quantité d'eau échangeable, est constitué par le bois d'aubier du tronc, des branches et des grosses racines (WARING \& RUNNING, op. cit.). La réserve hydrique y est très importante mais ne peut être mobilisée que dans des délais plus importants, de l'ordre de plusieurs jours au minimum. Ainsi, lors des séquences de dessèchements-réhydratations, le flux hydrique qui circule du sol vers l'atmosphère est tamponné par les variations de stock d'eau dans le réservoir-arbre, ce qui peut entraîner des divergences entre l'évapotranspiration réelle et la variation de l'humidité du sol.

Enfin, en ce qui concerne la méthode de mesure, non seulement elle permet de travailler avec précision et peu d'inertie au pas de temps horaire, mais aussi la faible dérive dans le temps des capteurs permet d'envisager leur utilisation à long terme pour estimer la transpiration d'un peuplement forestier. Ainsi, en 1984, certains capteurs ont fonctionné pendant six mois de façon continue et sans qu'apparaisse de biais significatif par rapport à l'index de transpiration maximale que nous avons défini au paragraphe 3.3. Grâce au faible coût et à la facilité de mise en place de cette méthode, il 
sera possible d'étudier la variabilité inter-arbres des transferts hydriques et d'estimer la transpiration des peuplements forestiers. Toutefois, les phénomènes de stockage et déstockage de l'eau dans les organes vivants situés au-dessus des capteurs de flux peuvent conduire à des écarts entre le flux de sève et la transpiration.

Reçu le 25 mai 1986.

Accepté le 11 septembre 1986.

\begin{abstract}
Summary
Sap flow measurements in Douglas-fir tree trunks by means of a new thermal method
\end{abstract}

Water transfers within Douglas-fir trunks are studied through a new method of sap flow measurement. Two trees growing in a forest stand were submitted to artificial drought by suppression of incident rainfall. There were no significant azimuthal differences between sap flow measured during 23 days (fig. 1), along three axis. Azimuthal differences were more important during sunny days, but were always less than $6 \mathrm{p}$. 100 . Sap flow were then measured at two different heights : buttress $(1 \mathrm{~m})$ and base of the living crown $(8 \mathrm{~m})$. For a well-watered tree, sap flow density was always greatest at the base of the crown. Total flows (estimated by product of sap flow density and sapwood cross sectional area) were however equal notwithstanding vertical level of measurement (see fig. 2). The decrease of sap flow during an induced drought was compared with a model of potential transpiration (see eq. 4). Reduction of transpiration starts when the mean water potential in the first $60 \mathrm{~cm}$ of soil was $-0,15 \mathrm{Mpa}$. Differences in total sap flow according to level of measurements increased progressively during the drought period (fig. 4). The difference between inflow at the lowest level $(1 \mathrm{~m})$ and outflow at the highest $(8 \mathrm{~m})$ represents the variation in the trunk water reserve (fig. 5), mainly located in the sapwood. Total water loss of 30 days amounted to approximately 28 liters, i.e. $4,2 \mathrm{~mm}$. The estimation of total water loss by the tree is 41 liters, i.e. $6,1 \mathrm{~mm}$. Such amounts of exchangeable water are reported in the litterature. Minimal predawn water potential decreased as low as - 1,25 MPa. Rehydration occured in two phases : first, there occured a refilling of living tissues, starting a few minutes after watering as shown at figure 6 . It also can be observed through a rapid increase of tree circumference. Thereafter, rehydration of the sapwood taked place progressively during 15 days following watering. Figure 5 shows a 4-5 days delay between watering and restoration of the sapwood water reserves. These observations confirm the theory of two water storage systems of differing capacities and time constants. storage.

Key words: Pseudotsuga menziesii, xylem sap flow, drought, temporal variations, water

\title{
Références bibliographiques
}

Aussenac G., Granier A., Ibrahim M., 1984. Influence du dessèchement du sol sur le fonctionnement hydrique et la croissance du Douglas (Pseudotsuga menziesii, Mirb. Franco). Acta oecologica, Oecol. plant., 5 (19), n” 3, 241-253.

Aussenac G., Granier A., Naud R., 1982. Influence d'une éclaircie sur la croissance et le bilan hydrique d'un jeune peuplement de Douglas (Pseudotsuga menziesii, Mirb. Franco). Can. J. For. res., 12 (2), 222-231.

Cohen Y., Kelliher F.M., Black T.A., 1985. Determination of sap flow in Douglas fir trees using the heat pulse technique. Can. J. For. res., 15, 422-428.

Granier A., 1981. Etude des relations entre la section du bois d'aubier et la masse foliaire chez le Douglas (Pseudotsuga menziesii, Mirb. Franco). Ann. Sci. for., 38 (4), 503-512. 
Granier A., 1985. Une nouvelle méthode pour la mesure du flux de sève brute dans le tronc des arbres. Ann. Sci. for., 42 (2), 81-88.

Greenidge K.N.H., 1957. Rates and patterns of moisture movement in trees. In : The Physiology of forest trees. Ed. K.V. Thimann.

Halloin S., Grip H., Jansson P.E., Lindgren A.. 19801. Micrometeorology and hydrology of pine forest ecosystem. "Theory and Models", in Structure and function of Northern coniferous forest. An ecosystem study. Ed. Persson, Ecoiogical bulletins, 32, 463-503.

Huber B., Schmidt E., 1937. Eine Kompensationsmethode zur thermoelecktrischen Messung langsamer Stafströme. Ber. dtsch. Bot. Ges., 55. 514-529.

JARvis P.G.. 1975. Water transfer in plants. In: Heai and Mass Transfer in the biosphere. I . Transfer processes in the plant environment. Ed. D.A. de Vries et N.H. Afgan.

Katerit N., Hallatre M., Perrier A., Durand R., 19:33. Transfert hydrique dans le végétal. 1 . Modélisation à l'échelle du couvert végétal en conditions naturelles. Oecol. Plant., 4, 11-26.

LASsole J.P., 1979. Stem dimensional fluctuations in Douglas-fir of different crown classes. For. Sci., 25, 132-144.

Lassoie J.P., Scott D.R.M., Fritschien L.J., 1977. Transpiration studies in Douglas-fir using the Heat Pulse Technique. For. Sci., 23 (3), 377-390.

Postlethwait S.N., Rogers B., 1958. Tracing the path of the transpiration stream in trees by the use of radioactive isotopes, Am. J. Bot., 45, 753-757.

SWANSON R.H., 1967. Seasonal course of transpiration of Lodgepole pine and Engelmann spruce. In Proc. International Symposium on Forest Hydrology, Penn. State Univ., 417-432 Pergamon Press, Oxford.

SWANSON R.H., 1974. Velocity distribution patterns in ascending xylem sap during transpiration. In : Flow its measurement and control in science and industry. Vol. I. Instrument society of America. Ed. Rodger B. Dowdell, 1425-1430.

VITE J.P., RUDINSKY J.A., 1959. The water-conducing systems in conifers and their importance to the distribution of trunk injected Chemicals. Bisyce Thompson Inst. Contrib, 20, 27-38.

WARING R.H., RUNNING S.W., 1978. Sapwood watcr storage : its contribution to transpiration and effect upon water conductance through the stems of old-growth Douglas-fir. Plant, Cell and Environment, 1, 131-140. 